\title{
DECAY AT INFINITY OF SOLUTIONS TO PARTIAL DIFFERENTIAL EQUATIONS WITH CONSTANT COEFFICIENTS
}

\author{
BY \\ WALTER LITTMAN(1)
}

1. Introduction. According to a classical result of Rellich [6] if $u$ is a solution to the Helmholtz equation

$$
\Delta u(x)+k^{2} u(x)=0
$$

in the exterior of a sphere, and if $u=o\left(|x|^{-(N-1) / 2}\right)$ as $x \rightarrow \infty$, where $N$ is the dimension of the space, then $u$ vanishes identically in that region. Numerous improvements of this result have been given, for example Miranker [5], Kato [2], Wilcox [9], Wolsson [10].

The above mentioned result can be restated in the following form: Suppose

$$
\Delta u+k^{2} u=f(x)
$$

and $f(x)$ is a $C^{\infty}$ function with compact support then $u$ must also have compact support, which by the unique continuation property must be the same as that of $f$.

More recently, Trèves [8] has investigated the following problem: Let $P(D) \equiv P((1 / i)(\partial / \partial x))$ be a partial differential operator with constant coefficients. If $P(D) u=f$, and $f$ in $C^{\infty}$ and has compact support, when can we conclude that $u$ has compact support, assuming that $u \in \mathscr{S}$, i.e. $u$ is infinitely differentiable and together with its derivatives decays at infinity faster than any power of $x^{-1}$ ? Trèves showed that for the answer to be in the affirmative it is necessary and sufficient that the complex solution manifold of each irreducible factor of the polynomial $P(x)$ intersect $R^{N}$.

It seems desirable to be able to reach the conclusions of Trèves' result without the strong condition that $u$ be rapidly decreasing at infinity, but with conditions at infinity more nearly like those in the Rellich result, i.e., decay faster than a prescibed power. For this weakening of hypothesis we must, however, pay a price i.e., one imposes greater restrictions on the polynomial $P$. That is the point of Theorem 1.

Received by the editors December 23, 1965.

(1) The author would like to express his thanks to Louis Nirenberg and Alfred Aeppli for encouragement and helpful advice in the writing of this paper. The work represented here was partially supported by USAF Grant-AFOSR 883-65. 
THEOREM 1. Let each irreducible factor $Q$ of the polynomial $P$ have the following properties

(a) the set $S$ of real solutions to $Q(x)=0$ forms a smooth $N-1$ dimensional surface $S$;

(b) $\operatorname{grad} P(x) \neq 0$ on $S$;

(c) at each point of $S, k$ of the $N-1$ principal curvatures are different from zero;

Suppose $u \in C^{\infty}\left(R^{n}\right)$ satisfies the equation

$$
P(D) u=f, \quad f \in \mathscr{D} ;
$$

and $u(x)=o\left(|x|^{k / 2-(N-1)}\right)$;

Then $u$ has compact support $\left({ }^{2}\right)$.

REMARK 1. By Lions' theorem on the support of a convolution, it follows that the convex hull of the support of $u$ is the same as the convex hull of the support of $f$. In case the equation $L u=0$ satisfies the unique continuation property, the supports are actually the same.

REMARK 2. Condition (a) of Theorem 1 implies that each irreducible factor $Q$ of $P$ must be real, apart from a multiplicative complex constant. To see this, let $Q=Q_{1}+i Q_{2}$. Picking a point $x_{0}$ on $S$, the complex solution manifolds of $Q_{1}$ and $Q_{2}$ through $x_{0}$ must each be $N-1$ complex dimensional. Their intersection $W$ must be either $N-2$ complex dimensional or $N-1$ complex dimensional. In the first case $W \cap R^{n}$ is $N-2$ real dimensional, contrary to assumption. In the second case $Q_{1}$ and $Q_{2}$ vanish simultaneously on an $N-1$ complex dimensional set, implying their ratio is constant. Thus we may without loss of generality assume that $P$, as well as each of its irreducible factors is real.

2. Proof of Theorem 1 modulo asymptotic estimates. We begin by introducing some notation and stating some preliminary results used in the proof, some of which are proved in later sections.

We shall use $L$ to denote the operator $P(D) . \mathscr{D}$ denotes the space of functions $C^{\infty}$ with compact support in $R^{n} . \mathscr{F}(\mathscr{D})$ shall denote the space of Fourier transforms of functions in $\mathscr{D}$. The Fourier transform $\hat{w}=\hat{w}(x)$ of the function $w(y)$ is given by

$$
w(x)=\left(\frac{1}{2 \pi}\right)^{N} \int e^{-i x \cdot y} w(y) d y .
$$

We shall often refer to $e^{i()}$ by $e()$. For $w \in \mathscr{F}(\mathscr{D})$ define the mapping

(2) Wolsson [10] derives this result for the special case in which the differential equation is elliptic, and in which $k$ has the value $N-1$ (corresponding to a surface $P(x)=0$ with only convex sheets). His derivation is based on a study of the behavior of the fundamental solution at infinity. Estimates for such fundamental solutions are also due to V.V. Grusin and B. B. Vainberg. (See [1] and references there.) 


$$
w \rightarrow v(y) \equiv T w \equiv \text { P.V. } \int e^{i x \cdot y} \frac{\hat{w}(x)}{P(x)} d x .
$$

Here "P.V." means "Cauchy principal value". The "Principal value" is discussed in $\$ 4$, and the above integral is studied in later sections.

One easily established property of the function $v$ as defined above is that it satisfies the equation

$$
P(D) v=w .
$$

Whe e there is no chance of confusion we shall let $r$ stand for either $|x|$ or $|y|$.

LeMma 1. For any $C^{\infty}$ functions, $u$, $v$, such that

$$
\begin{aligned}
D^{\alpha} v & =O\left(r^{-k / 2}\right), \quad|\alpha| \leqq m, \\
u & =o\left(r^{k / 2-(N-1)}\right),
\end{aligned}
$$

we have

$$
(L v, u)=(v, L u)
$$

(Parentheses denote the $L^{2}$ inner product.)

Proof. Let $\phi(x)=v(x) \psi_{h}(x)$, where $\psi_{h}(x) \equiv \psi(r-h)$, and where $\psi(t)$ is a $C^{\infty}$ function of the single real variable $t$ such that

$$
\begin{aligned}
\psi(t) & =1 & & \text { for } t \leqq 0, \\
& =0 & & \text { for } t \geqq 1 .
\end{aligned}
$$

We have

$$
L\left(v \psi_{h}\right)=L v \cdot \psi_{h}+\Sigma a_{\alpha \beta} D^{\alpha} v \cdot D^{\beta} \psi_{h},
$$

where the summation extends for $|\alpha|<m,|\beta|>0,|\alpha|+|\beta| \leqq m$. It follows that

$$
\begin{aligned}
(L(\phi)-L(v)) & =L v \cdot(1-\phi)+\Sigma a_{\alpha \beta} D^{\alpha} v \cdot D^{\beta} \psi_{h}, \\
(L(\phi-v), u) & =((1-\phi) L v, u)+\Sigma a_{\alpha \beta}\left(D^{\alpha} v \cdot D^{\beta} \psi_{h}, u\right) .
\end{aligned}
$$

Since the $D^{\beta} \psi_{h}$ are bounded it suffices to estimate

$$
\int_{h<r<h+1}\left|D^{\alpha} v\right||u| d x, \quad 0 \leqq|\alpha| \leqq m .
$$

This integral is majorized by

$$
o(1) \cdot r^{-k / 2} \cdot r^{k / 2-(N-1)} \cdot r^{(N-1)}=o(1) .
$$

Hence $(L \phi, u)-(L v, u) \rightarrow o$ as $h \rightarrow \infty$. But $(L \phi, u)=\left(v \psi_{h}, L u\right) \rightarrow(v, L u)$ as $h \rightarrow 0$, which is the desired result. 
LEMMA 2. Let $v$ be given by (2.1). Then

$$
D^{\alpha} v=O\left(r^{-k i 2}\right) \text { as } r \rightarrow \infty,
$$

uniformly with respect to all directions and any finite number of derivatives.

The proof is given in $\$ \S 5-7$.

LEMMA 3. Let $S$ be $C^{\infty} N-1$ surface embedded in $R^{N}$ and a smooth mass density $\alpha$ with compact support in $S$ be given on $S$. Suppose that at each point of $S, k$ of the $N-1$ principal curvatures are different from zero. Then

$$
I(y)=\int_{x \in S} e^{i x \cdot y} \alpha(x) d S_{x}=O\left(|y|^{-k / 2}\right) \text { as } y \rightarrow \infty .
$$

Furthermore any derivative $D^{\alpha} I(y)$ of fixed order satisfies the same condition.

The first part of the lemma is proved in [4]; the second part is an immediate consequence of the first.

We now proceed with the proof of Theorem 1.

Case I. Suppose $f \equiv 0$. Then by Lemmas 1 and $2,(w, u)=(T w, L u)=0$ for all $w \in \mathscr{F}(\mathscr{D})$, or $(w, u)=0$ for all $\hat{w}$ in $\mathscr{D}$. Hence $\hat{u} \equiv 0$ and $u \equiv 0$.

Case II. Suppose there exists a point in $R^{N}$ at which $P\left(x_{0}\right)=0$ and $\hat{f}\left(x_{0}\right) \neq 0$. Since $f$ has compact support $\hat{f}$ is analytic, hence continuous. Thus $\hat{f}(x) \neq 0$ in a whole open set intersecting $S$. Let $\alpha$ be a $C^{\infty}$ function on $S(\equiv P(x)=0)$ with compact support. Consider the measure $\mu \equiv \mu_{\alpha}$ with support on $P(x)=0$ defined by

$$
\int_{R^{N}} \phi(x) d \mu=\int_{S} \phi(x) \alpha\left(x_{S}\right) d S, \quad \phi \in \mathscr{D} .
$$

Clearly, for some such function $\alpha$ we have

$$
\int \hat{f}(x) d \mu_{\alpha} \neq 0 .
$$

Next, given $\hat{v}=(T w)^{\wedge}, w \in \mathscr{F}(\mathscr{D})$, let us determine a constant $a$ in such a way that i.e., $a=-(\hat{v}, \hat{f}) /(\mu, \hat{f})$.

$$
(\hat{v}+a \mu, \hat{f})=0 \text {, }
$$

With $a$ thus determined (and depending on $w$ ), define the transformation $T^{\prime}$ by

$$
\left(T^{\prime} w\right)^{\wedge}=\hat{v}+a \mu,
$$

i.e., $v^{\prime}(y)=T^{\prime} w(y)=v(y)+a \int e^{i x \cdot y} d \mu(x)$. It is easily seen that the integral is a solution to $L\left(\int\right)=0$, hence that $L v^{\prime}=w$. It follows from Lemmas 2 and 3 that $D^{\alpha} v^{\prime}=O\left(r^{-k / 2}\right)$. Hence, since $\left(v^{\prime}, f\right)=0$, by Lemma 1 we have

$$
0=\left(L v^{\prime}, u\right)=(w, u) \text { for all } w
$$

hence $\hat{u} \equiv 0$ and $u \equiv 0$. 
Case III. The remaining possibility is that $\hat{f}(x) \neq \equiv$ and $\hat{f}(x)$ vanishes on the $N-1$ surface $S \subset R^{N}$ where $P(x)=0$. We shall need the following lemma taken from Trèves [7].

LEMMA 4. Let $P\left(x_{1}, \cdots, x_{N}\right)$ be an irreducible polynomial with complex coefficients and let $V$ be the set of zeros of $P\left(z_{1}, \cdots, z_{N}\right)$ in $N$ dimensional complex space $C^{N}$. Let $\phi$ be an entire function on $C^{N}$. Assume that the function $\phi / P$ defined in $C^{N}-V$ can be extended as a holomorphic function to an open set $\Omega$ intersecting $V$. Then $\phi / P$ can be extended to $C^{N}$ as an entire function.

Suppose first that $P$ is irreducible. Pick a point $x_{0}$ on $S$. The set $V$ of complex zeros of $P$ restricted to a complex neighborhood $U$ of $x_{0}$ is an analytic set of complex dimension $N-1$. Since $f$ has compact support, $\hat{f}(x)$ can be extended as an entire function $\hat{f}(z)$ of exponential type. In $U$ (possibly restricted further) $\hat{f}(z)$ vanishes on a set of complex dimension $N-1$ containing $x_{0}$. Hence the set $W$ of common zeros of $P(z)$ and $\hat{f}(z)$ in $U$ either coincides with the set of zeros of $P(z)$ in $U$ or has complex dimension $N-2$. In the latter case however the restriction of $W$ to $R^{N}$ must have real dimension $\leqq N-2$, contrary to assumption. Hence whenever $P(z)$ vanishes in $U, \hat{f}(z)$ must also vanish. From this and the fact that $\operatorname{grad} P\left(z_{0}\right) \neq 0$ it follows that $\hat{f}(z) / P(z)$ can be continued as a holomorphic function to a complex neighborhood of $x_{0}$. Using the above lemma it follows that $\hat{u}_{1}(x)=\hat{f}(x) / P(x)$ can be extended to all of $C^{N}$ as an entire function. It is easily seen that this function must be of exponential type. This implies that $u_{1}(y)$ must have compact support. Hence, by case $\mathrm{I}$, so must $u(y)$.

Next suppose $P$ is not irreducible, say, for example $P=Q_{1} Q_{2}$ where $Q_{1}$ and $Q_{2}$ are irreducible. Since grad $P \neq 0$ on $S$, the real solution manifolds $S_{1}$ of $Q_{1}$ and $S_{2}$ of $Q_{2}$ must not intersect. Hence $\operatorname{grad} Q_{1} \neq 0$ on $S_{1}, \operatorname{grad} Q_{2} \neq 0$ on $S_{2}$, and $\hat{f} / Q_{2}$ can, as before, be extended as an entire function (since $\hat{f}$ vanishes where $Q_{2}$ does in $R^{N}$ ). We now repeat the argument, letting $\hat{f} / Q_{2}$ take the place of $\hat{f}$ and $Q_{1}$ replace $Q_{2}$. Clearly this argument can be extended to any number of factors.

3. Fourier transforms of surface-carried mass distributions. The purpose of this section is to state a previously proved result. Suppose we are given a $C^{\infty} N-1$ surface $S$ embedded in $R^{N}$. $S$ need not be a closed surface, i.e. $S$ may have a boundary, which however we do not include in $S$. Also given is a $C^{\infty}$ mass distribution $\alpha(x)$ defined on $S$ with compact support $S^{\prime}$ in $S$. At each point of $S$ the Gaussian curvature $K$, i.e. the product of $N-1$ principal curvatures, is bounded away from zero. Given a unit vector $\omega$, let $p_{j}=p_{j}(\omega)$ be the (finite set of) points on $S^{\prime}$ at which the normal to $S$ is parallel to $\omega$. Taking $\omega$ as the positive direction at $p_{j}$, denote by $k_{+}^{j} \equiv k_{+}^{j}(\omega)$ and $k_{-}$the number of positive and negative principal curvatures at $p_{j}$ respectively. Letting $n=N-1, y=r \omega(r>0), e^{i()}=e()$, and denoting surface element by $d S$, the following asymptotic formula holds as $r \rightarrow+\infty$, uniformly with respect to all directions $\omega$. 
$\int_{x \in S} e(x \cdot y) \alpha(x) d S_{x}$

$$
\begin{aligned}
= & r^{-n / 2} \sum_{j}\left(\frac{\pi}{2}\right)^{n / 2}(1+i)^{k^{j}+}(1-i)^{k^{j}-}\left|K\left(p_{j}\right)\right|^{-1 / 2} e\left(p_{j} \cdot y\right) \alpha\left(p_{j}\right) \\
& +O\left(r^{-(n / 2)-(1 / 2)}\right) .
\end{aligned}
$$

This formula is essentially derived in [4], where, however, it is not stated explicitly in this form. There only one point $p_{j}$ occurs, which is the origin, and the factors involving the $k$ 's are erroneously omitted (although that is of no further consequence either there or in the present paper). The essence of the proof is to show that the asymptotic behavior of the integral is the same (within a constant factor) as that of the integral $\int e\left(\lambda_{1} x_{1}^{2}+\lambda_{2} x_{2}^{2}+\cdots+\lambda_{n} x_{n}^{2}\right) d x_{1} \cdots d x_{n}$, the $\lambda$ 's being the principal curvatures.

It should be noted that for the case of closed convex $S$ a similar result has been obtained by Grušin [1], who also obtained estimates for integrals of the type (2.1) for that case. However his method does not seem suitable to yield (3.1) in the nonconvex situation.

4. The principal value integral. Let $A(x)$ and $g(x)$ be "sufficiently smooth", complex valued functions defined in $R^{N}, g$ with compact support. Let $S$ be the surface $A(x)=0$. It is assumed that $\operatorname{grad} A \neq 0$ on $S$ near the support of $g$, and that the support of $g$ is bounded away from the boundary of $S$. We wish to define the Cauchy principal value

$$
\text { P.V. } \int_{R^{N}} \frac{g(x)}{A(x)} d x .
$$

By using an appropriate partition of unity it is easily seen that the definition and proof of existence of the principal value can be reduced to the case where the support of $g$ is contained in a sphere of arbitrarily small prescribed radius with center on $S$. Let us introduce a new coordinate system in such a sphere, depending smoothly on the $x$ coordinates,

$$
\{t, u\}=\left\{t_{1}, t_{2}, \cdots, t_{n}, u\right\} \quad(n=N-1)
$$

such that $u=0$ corresponds to $x \in S$. Suppose that the Jacobian $J=\partial(x) / \partial(t, u)$ of the transformation is bounded away from zero and infinity; then $g(x)=g_{1}(t, u)$ and $A(x)=A_{1}(t, u)$. By Taylor's theorem for fixed $t$,

$$
A_{1}(t, u)=u a_{1}(t)+u^{2} a_{2}(t, u),
$$

where $a_{1}(0) \neq 0$ and $a_{1}$ and $a_{2}$ are smooth functions. Then

$$
\begin{aligned}
A(x)^{-1} & =\left[u a_{1}(t)+u^{2} a_{2}(t, u)\right]^{-1} \\
& =\left[u a_{1}(t)\right]^{-1}+R(t, u),
\end{aligned}
$$


defining $R$ as a smooth function of $t$ and $u$. We may, without loss of generality, suppose that the support of $g$ lies in the set $|u|<1$. Then

$$
\begin{aligned}
I_{\varepsilon} & =\int_{\varepsilon<|u|<1} g(x) / A(x) d x \\
& =\int g_{1}(t, u) \cdot J(t, u) A_{1}(t, u)^{-1} d t d u .
\end{aligned}
$$

Writing $g_{1} J \equiv g_{2}$ the above integral equals

$$
\begin{aligned}
I_{\varepsilon}= & \int_{\tau \in R^{n}} \int_{\varepsilon<|u|<1}\left\{\frac{g_{2}(t, 0)}{a_{1}(t) u} d u\right\} d t \\
& +\int_{\varepsilon<|u|<1} \frac{g_{2}(t, u)-g_{2}(t, 0)}{a_{1}(t) u} d u d t \\
& +\int_{\varepsilon<|u|<1} g_{2}(t, u) R(t, u) d u d t .
\end{aligned}
$$

The first integral vanishes, while the remaining integrals converge as $\varepsilon \rightarrow 0$, since the integrands are smooth functions of $t$ and $u$.

It is easily seen that the value of $I_{0}=\lim _{\varepsilon \rightarrow 0} I_{\varepsilon}$ is independent of the particular coordinate system $\{t, u\}$ chosen. Hence, to evaluate or estimate it one may choose the one most convenient. From here on we shall omit the "P.V."' in front of the integral sign.

5. Estimate of Fourier transforms: the almost horizontal part of the surface, $K \neq 0$. To prove Lemma 2 for the case $K \neq 0$, i.e., $k=N-1$, it is necessary to estimate the integral

$$
\int_{R^{N}} \frac{g(x)}{P(x)} e^{i x \cdot y} d x
$$

where $f \in \mathscr{D}$. We divide $g$ into $g_{1}$ vanishing near the surface $S: P(x)=0$ and $g_{2}$ with support near the surface $S$. By integrating by parts an appropriate number of times the integral involving $g_{1}$ is easily seen to approach zero as $y \rightarrow \infty$ faster than any negative power of $y$. Hence we may focus in $g_{2}$. We shall hereafter write $g$ for $g_{2}$.

We shall estimate in a given direction $\omega$. By means of a partition of unity, a translation and rotation of axes (which do not change the validity of the derived estimate), we may assume that $S$ passes through the origin, and that there it is perpendicular to the $y_{N}$ axis. For the time being we assume that the support of $g$ is contained in a sphere centered at the origin. We require the sphere to be sufficiently small so that in it the projection of the unit normal to $S$ on the $y_{N}$ axis exceeds $1 / 2$ in absolute value. We now introduce new coordinates $\{t, u\}$ as follows:

$$
t=\left\{t_{1}, t_{2}, \cdots, t_{n}\right\} \equiv\left\{x_{1}, x_{2}, \cdots, x_{n}\right\} \quad, \quad n=N-1 .
$$


If the surface $S$ near the origin is given by $x_{N}=z\left(x_{1}, \cdots, x_{n}\right)$, then the $u$ coordinate of the point $x=\left(x_{1}, \cdots, x_{N}\right)$ is given by $u=x_{n}-z\left(x_{1}, \cdots, x_{n}\right)$. Thus $u= \pm$ distance from $S$ measured in a direction parallel to the $x_{N}$ axis. (This coordinate transformation was used in [1].) The integral (5.1) is then given by the limit as $\varepsilon \rightarrow 0$ of

$$
I_{\varepsilon}=\int_{|u|>\varepsilon}\left[\int_{S . .} \frac{g_{1}(t, u)}{P_{1}(t, u)} J(t, u) e(y(z(t)+u)) d S_{u}\right] d u
$$

where $g(x)=g_{1}(t, u)$ etc., $S_{u}$ represents the surface $z\left(x_{1}, \cdots, x_{n}\right)=u, d S$ is surface element on it, and $J(t, u)$ is the appropriate Jacobian, which incidentally equals 1 at the origin. Let $g_{2}=g_{1} \cdot J$, and $\psi(u)$ be an even $C^{\infty}$ function of $u$ with compact support, having the property that $\psi(u) \equiv 1$ in an interval about the origin which is sufficiently large so that the set of $\{t, u\}$ where $\psi(u)=1$ contains the support of $g_{2}(t, u)$. Then

$$
\begin{array}{rlr}
I_{\varepsilon}= & \int_{|u|>\varepsilon} e^{i u y} \int_{S_{u}} e^{i z(t) y} g_{2}(t, u)\left[\left(u a_{1}(t)\right)^{-1}+R(t, u)\right] d S d u & \\
= & \int_{|u|>\varepsilon} \frac{e^{i u y}}{u} \psi(u) d u\left[\int_{S_{u}} e^{i z(t) y} g_{2}(t, 0) a_{1}(t)^{-1} d S\right] & \left(\equiv I_{1}(\varepsilon)\right) \\
& +\int_{|u|>\varepsilon} e^{i u y} d u \int_{S_{u}} \psi(u) e^{i z(t) y} \frac{g_{2}(t, u)-g_{2}(t, 0)}{u \cdot a_{1}(t)} d S & \left(\equiv I_{2}(\varepsilon)\right) \\
& +\int_{|u|>\varepsilon} e^{i u y} d u \int_{S_{u}} \psi(u) e^{i z(t) y} g_{2}(t, u) R(t, u) d t & \left(\equiv I_{3}(\varepsilon)\right) .
\end{array}
$$

Consider the integral $I_{1}^{\prime}$ in square brackets. Since the integrand is independent of $u$, clearly the $S_{u}$ may be replaced by $S=S_{0}$. From the results of $\S 3$ it follows that for $y>0$,

$$
I_{1}^{\prime}=M \cdot g_{2}(0,0) a_{1}^{-1}(0) y^{-n / 2}+y^{-n / 2-1 / 2} B(y),
$$

where $B(y)$ is a bounded function of $y$ and with the notation of $\S 3$,

$$
M=\left(\frac{\pi}{2}\right)^{n / 2}(1+i)^{k_{+}}(1-i)^{k-}|K|^{-1 / 2} .
$$

Since, as can be seen by integrating by parts $k$ times,

$$
\int_{-\infty}^{\infty} \frac{e^{i u y}}{u}(\psi(u)-1) d u=O\left(y^{-k}\right),
$$

we have $I_{1}(0)=\pi I_{1}^{\prime}+O\left(y^{-k}\right)$ for any $k>0$.

It is easily seen that $g_{2}(0,0)=g(0,0)$ and $a_{1}(0)$ is the normal derivative of $P$ with respect to $S$ at the origin. Next, letting $\varepsilon \rightarrow 0$ in $I_{2}(\varepsilon)$ and $I_{3}(\varepsilon)$ we see that 
the resulting integrals can be integrated by parts any number of times (since the inner integrands are smooth functions of $t, u)$, from which it follows that $I_{2}(0)$ and $I_{3}(0) \rightarrow 0$ faster than any power of $y^{-1}$ as $y \rightarrow \infty$.

Returning to the general situation we note that the set of points $p_{j}$ on $S$ at which $S$ is normal to a given direction is finite in number. By a partition of unity we can decompose $g$ into a sum of $g_{0}$ with support bounded away from the $p_{j}$ and $g_{j}$ with support in a neighborhood of $p_{j}$, but with support of different $g_{j}$ 's $(j \neq 0)$ bounded away from each other. We shall see later that the integral involving $g_{0} \rightarrow 0$ faster than any power of $1 / y$. Taking this for granted for the time being and reverting back to original coordinates, we have (in the notation of §3)

$$
\begin{aligned}
\int_{R^{N}} \frac{g(x)}{P(x)} e^{i x \cdot y} d x= & r^{-n / 2} \sum_{j} \pi \cdot\left(\frac{\pi}{2}\right)^{n / 2}(1+i)^{k^{j}+}(1-i)^{k^{j}-}\left|K\left(p_{j}\right)\right|^{-1 / 2} e\left(p_{j} \cdot y\right) g\left(p_{j}\right) \\
& \cdot\left[\frac{\partial P}{\partial \omega}\left(p_{j}\right)\right]^{-1}+O\left(r^{-\left(n^{\prime} 2\right)-(1 / 2)}\right),
\end{aligned}
$$

as $y \rightarrow \infty$ along the ray $y=r \omega, r>0, \omega$ being a unit vector. $\partial P / \partial \omega$ is the directional derivative of $P$ in the $\omega$ direction. That this formula is valid uniformly for all directions $\omega$ follows from a careful checking of the proof.

6. Estimate for nonhorizontal part of surface. In this section we estimate the integral (5.1) where the support of $g$ is near $S$ but bounded away from the set of points on $S$ at which $S$ is normal to $\omega$. We need not assume here that $K \neq 0$. By use of a partition of unity we see that it suffices to consider the case where the support of $g$ lies in a sphere of arbitrarily small, but fixed radius, such that in this sphere the unit normal to $S$ stays bounded away from the $\omega$ direction. We again translate and rotate axes so that $\omega$ coincides with the positive $y_{N}$ axis. We suppose that in the support of $g, S$ can be described by $x_{1}=Z\left(x_{2}, \cdots, x_{N}\right) \equiv Z(t)$, after a possible reordering of the $\left(x_{1}, \cdots, x_{N-1}\right)$. As before, we let $\psi(u)$ be an even $C^{\infty}$ function with compact support, having the property that $\psi(u) \equiv 1$ in an interval about the origin sufficiently large so that the set of $\{t, u\}$ where $\psi(u)=1$ contains the support of $g(t, u)$. Letting $u=x_{1}-Z\left(x_{2}, \cdots, x_{N}\right), g(x)=g_{1}(t, u)$, $g_{2}=J \cdot g_{1}$, as in $\S 5$, the integral (5.1) is the limit as $\varepsilon \rightarrow 0$ of the integral

$$
\begin{aligned}
\int_{|u|>\varepsilon} d u & \int_{S_{u}} \frac{g_{2}(t, u)}{P(t, u)} e^{i Z(t) y} d S_{u} \\
= & \int_{|u|>\varepsilon} \psi(u) \frac{d u}{u} \int_{S_{u}} \frac{g_{2}(t, 0)}{a_{1}(t)} e^{i Z(t) y} d S_{u} \\
& +\int_{|u|>\varepsilon} \int_{S_{u}} \frac{g_{2}(t, u)-g_{2}(t, 0)}{u a_{1}(t)} e^{i Z(t) y} \psi(u) d S_{u} d u \\
& +\int_{|u|>\varepsilon} \int_{S_{u}} e^{i Z y} g_{2}(t, u) R(t, u) \psi(u) d S_{u} d u .
\end{aligned}
$$


The first integral vanishes; letting $\varepsilon \rightarrow 0$, we notice that in the last two integrals the integrands are smooth, hence for fixed $u$ the inner surface integrals can be estimated by the results of [4] $\$ 2$, according to which they approach zero faster than any power of $y$ as $y \rightarrow \infty$, and this uniformly with respect to $u$. Hence the iterated integrals also approach zero in the same manner.

7. The case of zero curvature. We now consider the integral involving $g$ with support in a neighborhood of the set of points on $S$ at which the normal to $S$ points in the $\omega$ direction. It suffices to consider $g$ with support in a sufficiently small sphere with center on $S$, so that after translation and rotation of axes, the center of the sphere is the origin, $\omega$ points in the direction of the $x_{n+1} \equiv x_{N}$ axis, and the surface $S$ is described in the sphere, (after possibly renaming some of the $x$ 's among $x_{1}, \cdots, x_{n}$ ) by

$$
x_{N}=z\left(x_{1}, \cdots, x_{n}\right)=\sum_{j=1}^{k} \lambda_{j} x_{j}^{2}+O\left(|x|^{3}\right) .
$$

Let $x^{\prime}=\left(x_{1}, \cdots, x_{k}\right)$ and $x^{\prime \prime}=\left(x_{k+1}, \cdots, x_{n}\right)$. Consider the integral

$$
I=\int\left[\int \exp \left[i z\left(x^{\prime}, x^{\prime \prime}\right) y\right] \frac{g\left(x^{\prime}, x^{\prime \prime}\right)}{P\left(x^{\prime}, x^{\prime \prime}\right)} d x^{\prime}\right] d x^{\prime \prime} .
$$

Keeping $x^{\prime \prime}$ fixed, the inner integral $=O\left(y^{-k / 2}\right)$ as $y \rightarrow \infty$ uniformly in $x^{\prime \prime}$. Integrating with respect to $x^{\prime \prime}$ gives us

$$
I=O\left(y^{-k / 2}\right) \text { as } y \rightarrow \infty .
$$

Combining this result with results of $\S \S 5,6$ shows that

$$
h(y) \equiv \int_{R^{N}} \frac{g(x)}{P(x)} e^{i x \cdot y} d x=O\left(|y|^{-(k / 2)}\right) \quad \text { as } y \rightarrow \infty .
$$

That the result holds uniformly in all directions of the vector $y$, again follows from a careful analysis of the proof. That the same estimate is valid for any derivative $D^{\alpha} h(y)$ can be seen immediately from the formula

$$
D^{\alpha} h=\int \frac{x^{\alpha} g(x)}{P(x)} e^{i x \cdot y} d x,
$$

where $x^{\alpha}=x_{1}^{\alpha_{1}} x_{2}^{\alpha_{2}} \cdots x_{N}^{\alpha_{N}}$. This completes the proof of Lemma 2.

\section{Improvements and refinements.}

THEOREM 2. Let us suppose that for a certain polynomial $P(x)$ the hypothesis of Theorem 1 are not satisfied but that there exist a finite number of points such that if we delete closed neighborhoods of these points, no matter how small, from the surface $P(x)=0$ then in the remaining surface the conditions of Theorem 1 prevail; then the conclusions of Theorem 1 are still valid. 
To see this we need only change the proof of Theorem 1 slightly. Namely in selecting $\hat{w}(x) \in \mathscr{D}$, we choose the $\hat{w}$ so that it vanishes in said neighborhoods. In case II care must be taken to insure that the measure $\mu$ vanishes in these neighborhoods. This can be accomplished by appropriate choice of $\mu$ and by choosing the neighborhoods sufficiently small. It then follows (in Cases I and II) that $\hat{u}$ vanishes except at the above mentioned points. $\hat{u}$ must then be a sum of delta functions and derivatives of delta functions, which means that $u$ must violate the assumed growth estimate, unless $u$ vanishes identically. Case III proceeds as before.

EXAMPLE. The wave operator satisfies the conditions of Theorem 2 with $k=N-2$. Hence one must assume $u=o\left(r^{-N / 2}\right)$. The operator $\square u+u$, on the other hand satisfies the conditions of Theorem 1 with $k=N-1$. Hence it suffices to assume $u=o\left(r^{-(N-1) / 2}\right)$.

Secondly the proof of lemma 1 is also valid if one only assumes that the volume average of $u$ over the shell $r \leqq|x| \leqq r+1$ is $o\left(r^{k / 2-(N-1)}\right)$, instead of the uniform approach to zero.

Finally, instead of assuming $u \in C^{\infty}$, it suffices to assume that $u$ is locally integrable and is a generalized solution. This is seen by mollifying $u$ and applying our results to the mollified function.

\section{BIBLIOGRAPHY}

1. 1. V. V. Grušin, On Sommerfeld type conditions, Mat. Sb. (N. S.) 61 (103) (1963), 147-174. (Russian)

2. T. Kato, Growth properties of solutions of the reduced wave equation with a variable coefficient, Comm. Pure Appl. Math. 12 (1953), 403-425.

3. J. L. Lions, Supports dans la transformation de Laplace, J. Analyse Math. 2 (1953), 369-380.

4. W. Littman, Fourier transforms of surface-carried measures and differentiability of surface averages, Bull. Amer. Math. Soc. 69 (1963), 766-770.

5. W. L. Miranker, The reduced wave equation in a medium of variable index of refraction, Comm. Pure Appl. Math. 10 (1957), 491-502.

6. F. Rellich, Uber das asymptotische Verhalten der Lösungen von $\Delta u+k^{2} u=0$ in unendlichen Gebeiten, Jber. Deutsch. Math.-Verein. 53 (1943), 57-65.

7. F. Trèves, Lectures on partial differential equations with constant coefficients, Notas de Matemática No. 27, Instituto de Matemática Pura e Aplicada, Rio de Janeiro, 1961.

8. - Differential polynomials and decay at infinity, Bull. Amer. Math. Soc. 66 (1960), 184-186.

9. C. H. Wilcox, A generalization of theorems of Rellich and Atkinson, Proc. Amer. Math. Soc. 7 (1956), 271-276.

10. K. Wolsson, A generalization of the Rellich growth theorem, Thesis, New York University, 1962 (unpublished).

UNIVERSITY OF MiNNESOTA, MiNNEAPOLIS, MiNNESOTA 